Voix et Images

volxetimages

\title{
Narcisse et l'autre : pour un ethnotype québécois
}

\section{Heinz Weinmann}

Volume 3, numéro 2, décembre 1977

Victor-Lévy Beaulieu

URI : https://id.erudit.org/iderudit/200106ar

DOI : https://doi.org/10.7202/200106ar

Aller au sommaire du numéro

\section{Éditeur(s)}

Les Presses de l'Université du Québec

\section{ISSN}

0318-9201 (imprimé)

1705-933X (numérique)

Découvrir la revue

\section{Citer cet article}

Weinmann, H. (1977). Narcisse et l'autre : pour un ethnotype québécois. Voix et Images, 3(2), 266-276. https://doi.org/10.7202/200106ar d'utilisation que vous pouvez consulter en ligne.

https://apropos.erudit.org/fr/usagers/politique-dutilisation/ 


\section{Narcisse et l'autre: pour un ethnotype québécois}

'Puisqu'il est nécessaire que chacune des deux consciences de soi, qui s'opposent l'une à l'autre, s'efforce de se manifester et de s'affirmer, devant l'autre et pour l'autre, comme un être-pour-soi absolu, par là même celle qui a préféré la vie à la liberté, et qui se révèle impuissante à faire, par elle-même et pour assurer son indépendance, abstraction de sa réalité sensible présente, entre ainsi dans le rapport de servitude.

G. W. F. Hegel

Besides, she's she, and I'm I, and - oh dear, how puzzling it all is!

Lewis Carroll, Alice in Wonderland

L'identité canadienne est un miroir qui nous renvoie l'image de l'Autre quand nous nous y regardons.

Jean Bouthillette

\section{Psychologie individuelle et collective}

L'ethnopsychologie nous a montré que les peuples, au même titre que les individus connaissent des phases évolutives très marquées. Les peuples comme les individus ont leur "stade du miroir", passent par une crise d'adolescence avant d'atteindre la maturité pour finalement sombrer dans la sénilité. Ce parallèle de la psychologie collective avec la psychologie individuelle, pourtant, ne doit pas nous cacher les différences fondamentales entre les deux. Si pour un individu - sauf cas pathologiques l'évolution de maturation est un processus irréversible, une conscience nationale, moins homogène, plus fragile, puisque composée d'une infinité de consciences individuelles souvent divergentes, n'est jamais à l'abri de régressions qui mettent en cause l'acquis du processus de maturation anté- 
rieur. Ainsi par exemple, le sentiment de vieillesse d'un peuple peut faire place, par une sorte de retour inattendu, à un sentiment de jeunesse, de juvénilité: l' «automne du Moyen Âge», comme l'appelle si bien Huizinga, des $X I V^{\theta}$ et $X V^{\theta}$ siècles, avec son goût des danses macabres, cède la place à la «Renaissance», renouveau de l'Europe tout entière.

De plus, dans une étude ethnopsychologique, il faut tenir compte de l'interaction, pas forcément concordante entre l'évolution d'un peuple, d'une nation et d'un État. Par exemple, le peuple allemand s'est constitué au Moyen Âge, tandis que son existence en tant que nation et qu'État ne remonte qu'au XIX $X^{a}$ siècle. De cette dyschronie entre le cheminement de la notion de "peuple " d'une part et de "nation " et d' "État " d'autre part, naîtront de graves conflits d'identité qui aboutiront et se dénoueront dans la prise de pouvoir nazi, où, pour un moment, le peuple et la nation dans l'idéologie de «Blut une Boden», se confondront. L'Allemagne, en tant que nation, tragiquement, a connu sa crise d'adolescence, qui a mis en péril tout un héritage que le «peuple» a thésaurisé pendant un millénaire.

\section{Le drame de Narcisse ou le «mensonge romantique»}

Dans une telle perspective ethnopsychologique, le Québec présente un certain nombre de points communs avec l'Allemagne du XIX ${ }^{\theta}$ siècle. La comparaison entre les deux nous sera plus facile, une fois que nous aurons étudié le cas de l'Allemagne romantique. On le sait, c'est le romantisme qui, au début du $\mathrm{XIX}^{\ominus}$ siècle, de façon générale en Europe, est l'aiguillon de divers mouvements nationalistes: panslavisme, pangermanisme. Le romantisme, découverte jubilatoire de l'imago individuelle et collective dans sa totalité, c'est le «stade du miroir de ces nations». Rappelons à cet effet la définition de Jacques Lacan, théoricien de cette notion de "stade du miroir". "ll suffit de comprendre le stade du miroir comme une identification au sens plein que l'analyse donne à ce terme: à savoir la transformation produite chez le sujet quand il assume une irnage - dont la prédestination à cet effet est suffisamment indiquée par l'usage, dans la théorie, du terme antique d'imago [...]. Ce stade [...] nous paraîtra dès lors manifester en une situation exemplaire la matrice où le je se précipite en une forme primordiale, avant qu'il ne s'objective dans la dialectique de l'identification a l'autre ${ }^{1}$ et que le langage ne lui restitue dans l'universel sa fonction de sujet." (Écrits) Si le stade du miroir, selon Lacan, est un moment capital, le plus décisif, tout au moins en ce qui concerne la fixation de l'image de soi, il doit aussi être dépassé, sous peine de reproduire à l'infini la tautologie imagée de nous-mêmes. C'est le drame de Narcisse. Justement, le mythe de Narcisse, à travers les différents thèmes qui le modulent (double, scission du moi, reflet perdu, etc.) est un mythe typiquement romantique. Les œuvres d'un E.T.A. Hoffmann (les Aventures de la Saint-Sylvestre), d'un Albert de Chamisso (Peter Schlemihl), d'un Heinrich Kleist (Sur le théâtre de marionnettes), pour ne nommer que ceux-là, témoignent de l'emprise de Narcisse sur l'imagination romantique. Dans tous ces récits, l'autre $d u$ moi (reflet-ombre), à jamais impréhensible dans 
son évanescence même, se confond avec le reste du monde. Le destin de Narcisse se scelle dans cette unique image, reproduite ad libitum, qui fusionne de façon inextricable le Moi, l'Autre et le Monde. Il s'agit d'une confusion plutôt que d'une fusion, puisque Narcisse ne pourra jamais déployer dialectiquement ces trois éléments en tant que «moments" distincts, étant donné qu'au début est le “mensonge romantique " et narcissique de l'identité sur l'altérité. "ll se désire dans son ignorance luimême " lit-on déjà à propos de Narcisse dans les Métamorphoses d'Ovide. Désir pervers que celui de Narcisse, attisé par son ignorance quant à son véritable objet d'amour. "ll se prend pour un autre» voilà, vulgairement parlant, décrite la situation de Narcisse. Comme tout désir pervers, celui de Narcisse ne. pourra vraiment s'assouvir, fût-ce par la répétition obsessive d'une même situation, d'une même image, toujours la même: Narcisse est le mythe du Même. Écho, l'Autre, la Différence, se mue avec Narcisse en Égo. L'égo narcissique projetant son image sur le monde, il pourra ainsi confondre l'infinité réelle de ce dernier avec sa propre infinité imaginaire. Ainsi par exemple, dans les Discours sur la religion ${ }^{2}$ du philosophe romantique Schleiermacher, qui ne pourra pourtant être suspecté d'un narcissisme primaire comme certains poètes romantiques, nous lisons: “Aussi souvent que je tourne mon regard vers mon être intime, j'entre dans le domaine de l'infini, je contemple l'action de l'esprit que rien au monde, que jamais rien ne pourra détruire puisque c'est lui qui a créé le monde et le temps ${ }^{3}$." Ce n'est donc pas un hasard si un Pascal, aux antipodes du romantisme, découvreur de l'infini ontologique et mathématique, se méfie tant de l'analyse du moi 4 . Son fameux "le silence éternel des espaces infinis m'effraie" trouve son pendant logique dans son «le moi est haïssable ", car le regard narcissique, comme nous le montre Schleiermacher, en tournant le dos au monde ouvre seulement vers l'intérieur la béance des "espaces infinis" et inaugure ce que Freud appellera plus tard l'«analyse infinie ${ }^{5}$ ".

C'est encore en termes d' "infinité" et de "liberté" que Hegel dans son diagnostic impitoyable du romantisme caractérisera ce dernier. "L'esprit acquiert ainsi la conscience d'avoir en lui-même son «autre", son existence en tant qu'esprit et de jouir ainsi de son infinité et de sa liberté. Cette élévation de l'esprit vers lui-même, grâce à laquelle il trouve en lui-même son objectivité qu'il avait été obligé jusqu'alors de chercher dans le monde sensible et extérieur, et grâce à laquelle il acquiert le sentiment et la conscience de son union avec lui-même, constitue le principe fondamental de l'art romantique" (Cours sur l'Esthétique, chap. "L'art romantique"; nous soulignons $\left.{ }^{6}\right)$. Cette infinité et cette liberté de l'intériorité narcissique et romantique sont évidemment la contrepartie de la suppression de l'"Autre", de la phénoménalité extérieure qui ne sont même pas "relevées" (au sens hégélien de "aufgehoben"), mais tout simplement évacuées de l'intériorité.

Paradoxalement, selon Hegel, le romantisme inaugure aussi le réalisme, puisque le romantique ne voit plus de lien nécessaire entre son inté- 
riorité et son expression, sa représentation, son objectivation. Si tout exprime toujours cette intériorité, n'importe quel fait extérieur, n'importe quel accident l'illustrera. Plus besoin de maquiller, de styliser, de "naturaliser" le monde extérieur, la "réalité ", comme ce fut le cas pour l'art classique. "Aussi bien l'intériorité romantique peut-elle se manifester dans toutes les circonstances possibles et imaginables, s'accommoder de n'importe quels états et situations, commettre d'innombrables erreurs et s'engager dans des complications infinies, provoquer des conflits et se procurer des satisfactions de toute sorte, étant donné que ce qu'elle recherche, ce n'est pas un contenu objectif et valable en soi, mais son propre reflet, quel que soit le miroir qui le lui renvoie. " (lbid., nous soulignons.) On aura compris que le romantisme pour Hegel ne se limite pas au mouvement littéraire du début du $\mathrm{XIX}^{e}$ siècle, mais une vision du monde (Weltanschauung) qui naît au Moyen Åge et se dissout avec le romantisme, après y avoir atteint son apogée. (Sur toute la question de l'attitude de Hegel face au romantisme, voir Ph. Lacoue-Labarthe, «l'Imprésentable», in Poétique, $n^{\circ} 21,1975$, p. 53-86.)

\section{De la négation à la détermination: la définition}

L'Autre est la rive sur laquelle l'océan de l'infinité intérieure échoue, limite où elle finit, frontière qui la dé-finit. Car le problème de Narcisse en est un essentiellement de dé-finition. Le Narcisse gidien l'a bien compris, c'est pourquoi il a cessé de l'être. «A force de les contempler, il ne se distingue plus de ces choses: ne pas savoir où l'on s'arrête - ne pas savoir jusqu'où l'on va." (Le Traité du Narcisse) Narcisse en rejetant, en supprimant l'Autre restera l'éternel indéfini, puisqu'il supprime par là la frontière contre laquelle il puisse se définir. C'est pourquoi son discours sur la définition, c'est-à-dire son auto-analyse interminable, lui tiendra lieu de véritable dé-finition. II oublie que Je m'arrête où l'Autre commence. On ne s'étonnera donc pas que dans la philosophie hégélienne, philosophie de la limite, l'Altérité, l'Autre jouent un rôle capital. Sa philosophie ne serait-elle pas un ultime effort pour exorciser les ef-fusions romantiques qu'il a connues au "Stift" de Tübingen, où il se lie d'amitié avec Schelling et Hölderlin? A léna, où il sera Privatdozent de 1801 à 1807, il assistera à l'effervescence du groupe romantique d'léna qui s'assemble autour des frères Schlegel et qui compte parmi ses membres Novalis et Tieck. Plus qu'une simple coïncidence, la publication de sa Phénoménologie de l'esprit se fait la même année que son départ d'léna: elle consomme la rupture avec Schelling et avec l'esprit romantique.

Chez Hegel donc "limite", "finitude», détermination" sont synonymes. Il écrit ainsi dans une esquisse de la Science de la logique: "Quelque chose qui est defini a un rapport avec autre chose. L' «autre» est un être défini comme le non-être de ce quelque chose. Par conséquent, il a une borne, une limite, et il est fini. Ce qu'un quelque chose doit être en soi est appelé sa détermination." (Nous soulignons) Autrement dit, la conscience de soi, l'identique, pour sortir de son abstraction formelle vide 
de tout contenu, qui se résume à Je $=J e$, doit se poser par et dans une négation. La négation est le début d'une position, d'une détermination. Spinoza.l'avait formulé avant Hegel. Pour cette raison Hegel reconnaîtra sa dette à ce premier. "Spinoza est un point crucial dans la philosophie moderne. L'alternative est: Spinoza ou pas de philosophie. Spinoza établit le grand principe: “Toute détermination est une négation. " Le déterminé est le fini; or on peut montrer que tout, y compris la pensée [...] est un déterminé, renferme une négation; son essence repose sur la négation. " (Cours d'histoire de la philosophie) Évidemment, Hegel mesure la portée de Spinoza ici seulement par rapport à l'histoire de la philosophie. Pour notre propos, nous pouvons aller plus loin et dire: spinozisme ou pas d'identité!

II serait sans doute intéressant, mais cela dépasserait le cadre de cet article, de faire l'historique de cette négativité comme moteur du processus d'identification individuel et collectif, en commençant par la Renaissance. On l'a souvent dit, la Renaissance est le berceau de l'individualisme moderne. Or, l'individu, in-divis, s'affirme et se reconnaît dans la négation des autres, qui le limitent, le différencient. L'individu est dans la Différence. Ce n'est donc pas un hasard si, à la même époque, sur le plan collectif, on voit naître divers nationalismes et progressivement s'élaborer la notion d'État. Plus récemment, face à un ennemi commun, Napoléon, qui marquera les limites de l'Autre contre qui les nationalismes allemands pourront se définir en tant que nation. Fichte prononcera ses Discours à la nation allemande (1807). De la même façon, l'idée de l'Europe s'est constituée grâce aux grands voyages, aux circumnavigations de la terre. En s'éloignant, en partant, on a vu les limites du pays natal, de l'Europe. De la même façon qu'aujourd'hui, avec les voyages interplanétaires, une conscience planétaire commence à se développer. (Voir les études du Club de Rome.) C'est sur la lune que l'homme a découvert à la fois que sa Terre était belle et menacée. Les grands explorateurs Magellan, Cooke, Bougainville, nomment, désignent les pays, les choses qu'ils y rencontrent parce qu'ils devront les quitter. Nous touchons là une des lois fondamentales de la communication, du langage, qui donc en dernière analyse n'échappe pas non plus au travail de la négativité. Nommer une chose, c'est nier le “non-être de ce quelque chose", pour reprendre les termes mêmes de Hegel. II fallait attendre un Saussure pour que soit vraiment mis en évidence l' "opposivité " comme loi du fonctionnement du langage.

\section{Le travail de la conscience : reconnaissance et suppression de l'Autre}

Le travail du langage, de l'imaginaire, de la conscience de soi sont isomorphes. C'est en effet dans ces termes que Hegel explique le travail de la conscience de soi: «Ainsi cette conscience exerce une double activité:

1. pour supprimer l'altérité des objets et pour les poser comme égaux à elle-même,

2. pour sortir d'elle-même et se donner de la sorte objectalité et réalité présente. » Propédeutique philosophique 
Ces deux activités n'en font qu'une seule. (Dans la Propédeutique philosophique, nous lisons aussi plus loin: "Les deux aspects de la conscience de soi, celui qui pose et celui qui supprime, sont donc liés l'un à l'autre de façon immédiate. La conscience de soi se pose par la négation de l'altérité et elle est conscience pratique.") La conscience de soi s'affirme donc dans une double activité, mais qui est fondamentalement la même. Elle supprime l'altérité; ce qui bien sûr ne veut pas dire que l'autre disparaît totalement, sans laisser de trace - c'est le cas de Narcisse dans son horizon de conscience. Au contraire, la présence de l'autre est tout d'abord reconnue, elle est une réalité, non pas un fantasme, ni un fantôme; c'est justement elle qui au préalable dé-finit, dé-limite la conscience de soi. Ensuite, dans la suppression de l'autre (Aufhebung au sens hégélien $\mathrm{du}$ mot $=$ suppression et conservation), la conscience peut revenir à elle, enrichie de la connaissance de l'autre. L'Aufhebung est d'autre part le moment nécessaire de la conscience pour pouvoir se poser égale à autrui, après s'être mesuré avec lui. Enfin, la conscience de soi gagne son objectalité, en sortant d'elle, en se quittant. Mais cette objectivation de la conscience ne peut se faire qu'à partir de la reconnaissance de soi par autrui, reconnaissance de ma liberté. "En face de l'autre, chacun est absolument pour lui-même et singulier, et il exige en outre, d'être têl pour l'autre et d'être tenu pour tel par l'autre, d'avoir dans l'autre intuition de sa propre liberté comme liberté d'un étant-en-soi, c'est-à-dire d'être reconnu par l'autre." (Propédeutique philosophique) Est esquissée ici la fameuse dialectique du maître et de l'esclave, développée dans la Phénoménologie, et qui devient ici, dans le processsus de la conscience de soi, la dialectique de «maîtrise-servitude" ou de «service-obéissance».

\section{Le Québec et son Autre}

Le problème de l'identité, nous l'avons vu, est avant tout un problème de définition, de délimitation, au sens d'une confrontation avec l'Altérité. La question n'est pas tant de savoir comment je me définis, mais contre qui je me définis. Car mon regard posé sur moi se perd dans l'infini jeu de miroirs, qui ne mirent finalement qu'une conscience vide d'ellemême. Mais c'est justement le caractère fantômatique et fantasmatique de sa conscience qui fascine Narcisse: c'est sa réalité. Son reflet est son autre. Par là il évite la confrontation, la lutte (au sens de l'antagon grec) avec l'Autre.

Jean Bouthillette a montré ${ }^{7}$ comment la lutte, la confrontation avec l'Autre ont été esquivées au Québec depuis la bataille des Plaines d'Abraham. Depuis, seulement des escarmouches (en 1837), des actes isolés de "desperados" (pendant les années 1960) prennent sporadiquement l'Autre ouvertement comme cible de leur attaque. Mais dans la conscience collective la confrontation est désamorcée dans le grand étouffoir de la Constitution de 1867 qui délaie le concret dans l'abstrait, le particulier dans le général et l'opposition dans la conciliation. En effet, la Constitution de 1867, juridiquement, et pour la forme seulement, associe le vainqueur et 
le vaincu d'hier au sein d'une même confédération. Droits égaux pour tous! Cette générosité de l'Autre, fût-elle légale et abstraite, étouffe, mieux, désarme jusqu'à toute velléité de haine et d'agressivité contre lui. Cette agressivité potentielle qui ne peut plus se fixer, s'objectiver sur un ennemi, un Autre extérieur reflue finalement sur elle-même pour se décharger en un masochisme culpabilisant. L'ennemi d'antan, tel Protée, devenu insaisissable dans le sỳstème abstrait de la loi, partout et nulle part, se déréalise. Non, l'ennemi ce n'est plus l'Autre, c'est soi-même! "Notre inconscient refus de l'Anglais s'abreuve donc de cette haine issue de la Conquête. Mais comme pour la Conquête, nous avons «oublié " la haine, qui s'est tapie en nous comme une bête. Car elle est toujours là, arraisonnée en apparence mais qui nous dévore sans que nous le sachions et nous paralyse face à l'Anglais contemporain, qui est compatriote, un Canadien comme nous. Comment haïr cet Anglais qui n'a plus rien d'un soldat de Wolfe? " (Ibid., p. 71)

C'est là en 1867 que se consomme au Québec ce processus d'intériorisation - et d'infériorisation - commencé avec la Conquête. Le Québécois devient "romantique" au sens hégélien, puisque, pour rappeler sa définition, "l'esprit acquiert ainsi la conscience d'avoir en lui son autre... et de jouir ainsi de son infinité et de sa liberté». Infinité et liberté sans fond et donc sans fondement objectif, puisqu'ils s'irréalisent dans les profondeurs abyssales de l'océan intérieur que dorénavant nul rivage extérieur n'arrête plus, donc ne dé-finit plus. L'Église finalement confirme et canalise cette intériorisation en faisant luire dans le lointain la promesse d'une liberté infinie et éternelle. Liberté d'autant plus parfaite que l'individu et la collectivité se détournent de qui justement risque de les restreindre: l'Autre et la Réalité. L'Autre, l'ennemi, grâce à l'absolution générale qu'il reçoit au nom de la charité chrétienne se mue en "notre prochain."

Mais l'ennemi ainsi disparu dans le ciel des béatitudes chrétiennes aura un substitut inerte qu'il faut combattre comme son pire ennemi: la Matière. Elle a l'heureux avantage d'être partout, en nous et en dehors de nous. La lutte risque donc d'être épuisante et interminable. “ L'Esprit évacué de la matière se posait au principe de notre innocence. S'il survenait un grand malheur, ou un simple revers, c'est que l'esprit avait buté contre la matière coupable. Pour l'exorciser, il fallait la nier toujours davantage." (lbid., p. 74-75) Aux inconsolables qui ne pouvaient admettre avec gaieté de cœur un tel rejet de la matière, de la réalité, on promettait une peau de chagrin céleste qui, miraculeusement, s'agrandissait au fur et à mesure que la terrienne, la québécoise, se retrécissait. C'est le scénario du pari pascalien: «on hasarde avec certitude (matière - réalité) pour gagner avec incertitude" (l'intériorité, le royaume du ciel). Cette négation de la matière ne peut être poussée impunément à l'infini : à force de repousser la Matière, on finit pas hypostasier une matière. Non pas la matière moderne, celle de la Conquête, la technique, l'économie: elle est entre les mains de l'Autre. Matière archaïque, biblique, matière de subsistance, lais- 
sée vacante par l'ennemi: la glèbe, la Terre. Et encore, l'agriculturisme montrera au Québécois qu'on ne travaille pas la terre pour en jouir et pour la faire produire le plus possible, mais finalement pour se mortifier du péché d'avoir remué la terre, qui, ne l'oublions pas, reste matière et donc "mauvaise". Ne cultivons alors surtout pas les sols les plus fertiles, les plus faciles! Déboisons des terres non arables, cultivons des sols arides et caillouteux! «Et plus le sol est dur, plus il est méritoire de souffrir pour le faire produire. Or, il ne produit pas. Nous ne nous poserons pas de questions sur la qualité du sol: c'est nous qui ne sommes pas assez purs. La colonisation, c'est cette ascèse de l'innocence poussée jusqu'à la flagellation pour punir la matière coupable. Expier est notre vocation dans ce monde trop matériel. " (lbid., p. 75)

On l'aura remarqué, cette intériorisation qu'accompagne la négation de l'Autre et de la réalité dans l'analyse de Jean Bouthillette n'a rien à voir - sauf le nom - avec la négation comme négativité au sens hégélien. Pour éviter toute confusion, précisons que la première relève davantage de la dénégation au sens psychanalytique, au sens freudien du terme, et se trouve ainsi aux antipodes de la négation hégélienne même. Nous avons vu que "Authebung" (Derrida propose justement "relève" comme traduction pour éviter les équivoques du terme "suppression") loin de se réduire à une suppression pure et simple de l'Autre, pose l'Autre comme l'égal du sujet et, de ce fait, se reconnaît l'égal de l'Autre. Or rien de tel dans la dénégation narcissique de la conscience québécoise. Par sa dénégation de l'Autre, cette dernière fait de l'Anglais un fantasme qui la hante tout au plus comme un ressentiment («maudit Anglais»), mais qu'on ne rencontre plus dans le champ de la réalité pour l'affronter.

Que l'Autre occupe le champ réel (économique, technique), laissé vacant par le sujet québécois, après tout n'a pas de quoi nous étonner. Mais qu'il investisse également, subrepticement, sa conscience et son inconscient, voilà qui est plus grave. Et il semble que Hegel lui-même, tout au moins dans son analyse du romantisme, n'ait pas assez tenu compte de cette force pénétrante du regard de l'Autre qui impose, sans que le sujet s'en doute, sa “vision du monde», détruisant ainsi «son infinité et sa liberté " intérieures. Le sujet, vide de sa substance intime, de sa différence, perd totalement la notion de limite, de frontière (sa dé-finition) qui le sépare de l'Autre. Ainsi le monopole de l'Autre consacre le règne de l'assimilation $^{8}$ et de l'aliénation, au sens primitif du terme: l'alienus, (l'Étranger, l'Autre, l'Ennemi) me définit. Ironie du sort, Narcisse meurt par cela même qui l'a fait naître: son regard. Son ombre, son reflet, son irréalité avalent son être réel. "C'est à ce point de rencontre du système et de notre condition que se dévoile un renversement psychique fatal: nous nous voyons avec les yeux de l'Autre'. " (Ibid., p. 45)

\section{L'Écho de la voix}

Reste alors encore la voix de Narcisse, ou plutôt son écho qui retentit longtemps après que sa voix se soit tue. Ce qui le fascine dans sa voix, 
c'est comme dans son regard, l'immatérialité et le renvoi, le «feedback" de lui-même. Par sa voix, il parodie, il singe seulement l'Altérité puisqu'Écho, son Autre réel, Narcisse l'a tuée à force de la nier. La voix, et tout ce dont elle se fait porteuse, la langue, lui tiennent lieu de réalité: nommer le monde c'est déjà le posséder, évoquer la chose, c'est comme si on l'avait déjà. Se créent ainsi une série de satisfactions substitutives imaginaires et verbales d'autant plus jouissives qu'elles s'obtiennent immédiatement (elles ne dépendent que de moi!) sans ce détour laborieux et frustrant qui passe par la réalité. Certes, il ne s'agit pas de nier le rôle primordial de la langue comme outil de domination et de maîtrise du monde. Mais lorsque la langue cesse d'être un simple signe, un simple renvoi, lorsqu'elle devient un substitut de la réalité, elle perd nécessairement sa fonction médiatrice entre l'intérieur et l'extérieur, la subjectivité et l'objectivité, entre, en termes freudiens, le principe de plaisir et le principe de réalité.

Jadis, Hubert Aquin dans "Profession: Écrivain" a admirablement étudié - sous l'angle de la dialectique dominant/dominé, qui fut celle de Parti pris - cette survalorisation ici du symbolique, du littéraire, de la voix, qui doivent compenser les pertes au niveau de la réalité. Cessons, dit-il, de vivre “un roman écrit à l'avance ${ }^{10}$ ", cessons d'écrire tant que la réalité politique n'a pas acquis son propre poids, afin que les productions symboliques ne puissent entrer en concurrence avec les productions réelles (politiques et économiques). Tout le chassé-croisé entre la réalité et la fiction de la structure de Prochain Épisode s'annonce déjà en filigrane. Roman «interminable» (comme l'analyse «infinie = interminable» de Freud et de Narcisse) tant que la réalité politique ou la révolution n'a pas délivré l'imaginaire du flou des possibles intérieurs. "Quelque chose d'autre m'importe, un au-delà littéraire qui n'est pas une métalittérature, ni un nouveau déguisement de la vieille ambition, mais qui est la destruction du conditionnement historique qui fait de moi un dominé. En rejetant la domination, je refuse la littérature, pain par excellence des dominés, production symbolique dont on cède le monopole au dominé ce qui entraîne inévitablement une surproduction. N'a-t-on pas constaté que dans les pays colonisés se manifestait invariablement une surproduction littéraire? À défaut de réalité, on surproduit des symboles. " ("Profession: Écrivain », p. 52)

Ce parti pris symbolique ou nominaliste dénoncé par Hubert Aquin se retrouve justement dans l'étude par ailleurs très perspicace de Jean Bouthillette. Pourtant, il a été à bonne école, puisqu'il a réalisé une entrevue retentissante avec l'auteur de Prochain Épisode, "Écrivain, faute d'être banquier ${ }^{11}$ ". Pour Jean Bouthillette, tout le malheur du Canada français se réduit à une question de nom. L'Anglais s'étant insinué au Canada, il s'est emparé du nom de "Canadien" qui seul donnait une identité à l'habitant de descendance française en Amérique du Nord. «Nous étions Canadiens. Après 1760, l'Anglais en vient lui aussi à se dire Canadien. Non seulement s'empare-t-il de notre pays, mais il s'approprie notre nom de peuple, qui se charge de sa présence. " (Op. cit., p. 23) L'ancien Canadien qui collait à ce 
nom doit se dédoubler, se diviser en Canadien français pour ainsi se démarquer de l'Autre qui est venu "partager" son nom avec lui. «Par relation, notre langue peut ainsi être distinguée d'une identité canadienne qui en comprend deux, mais elle ne se distingue pas réellement en nous. Au sein d'un nom commun, la langue française nous distingue de l'Anglais, non de nous-mêmes. Nous ne sommes pas Canadiens plus quelque chose qui s'appelle la langue française, mais indistinctement Canadienfrançais. C'est en étant de langue française que nous sommes naturellement Canadiens, sans distance entre l'identité et la langue, qui forment un tout homogène. " (lbid., p. 29)

Ce qui a été vrai pour la Conquête, le sera aussi pour la "Reconquête", dernier chapitre du livre, le plus court, ne comportant que deux pages. Visiblement plus occupé à palper la «blessure toujours ouverte» ( $p$. 13) du passé, l'auteur «oublie» de penser à sa guérison possible dans l'avenir.Un seul mot, un seul nom va faire l'office rédempteur de la libération et de la reconquête. "A l'heure de la décolonisation du monde, cet instinct nous rend universels d'emblée. C'est lui qui, depuis 1960, nous fait lentement renaître à nous-mêmes ế au monde; c'est lui qui, dans l'intuition d'un nom - puisque tout a commencé dans un nom - retrouve dans toute sa réalité notre véritable identité, un nom qui lève toute ambiguïté, un nom clair et transparent, précis et dur, un nom qui nous reconstitue concrètement dans notre souveraineté et nous réconcilie avec nousmêmes: Québécois. " (Ibid., p. 95-96; nous soulignons.) Ce nom encore une fois ne renvoie pas à la réalité, il est sa propre réalité. Nom qui se dit "dur" et "concret" comme une chose palpable. Mais ne nous y trompons pas, ce nom n'est nul autre que l'écho de la voix de Narcisse qui va en répétant un seul nom: Narcisse.

\section{VII. Épilogue 1977}

Pas de Reconsquista spectaculaire et prolongée, ponctuée du son des canons et des mitraillettes. (Celle de l'Espagne avait duré 800 ans !) Le destin politique du Québec, comme celui de Narcisse, se scelle, par la voix: les voix muettes qu'émettent les urnes. L'issue du référendum dépendra d'un mot: indépendance, association, souveraineté, région? Le premier acte politique important du premier ministre québécois est un discours: à New York. La première loi (projet de loi $n^{\circ} 1$ ) est une loi sur la langue: l'art de faire du réel avec du symbolique.

Le Québec a pavoisé le 15 novembre: c'est normal. Mais il n'a pas mis ses drapeaux en berne pour la mort d'Hubert Aquin. Contretemps ironique que cette mort de quelqu'un pour qui le salut individuel fut toujours indissolublement lié au destin collectif et national. Au moment précis où le Québec troque le symbolique contre le réel (le pouvoir), Hubert Aquin opte définitivement pour le symbole par excellence, le suicide, qui scelle l'échec devant la réalité. Cette mort aura l'ambiguïté de son œuvre. Échec 
personnel? Ou l'écho des voix du 15 novembre lui a-t-il murmuré qu'on ne reconquiert pas son pays avec des voix?

automne 1975 - automne 1977

Heinz Weinmann

Collège de Rosemont

1. Nous soulignons.

2. Les Discours sur la religion datent de 1799 et sont le résultat de méditations poursuivies avec Frédéric Schlegel sur la philosophie de Spinoza.

3. Ce passage, d'inspiration fichtéenne, annonce déjà le "monde comme représentation " de Schopenhauer.

4. Sur la question de l'infini et des nombres en général, voir l'essai intelligent de Jacques Dufresne, Le 100,000ème exemplaire, essai sur la magie du nombre, Montréal, Éditions du Jour, 1975; plus particulièrement en ce qui concerne Pascal et l'infini p. 153 et sq.

5. Freud fait la distinction entre "analyse finie" et "analyse infinie", "endliche und unendliche Analyse ", traduit souvent prosaïquement par "analyse terminable" et " analyse interminable".

6. Voir aussi, «pour le romantisme, c'est le sujet individuel, réel animé d'une vie intérieure, qui acquiert une valeur infinie, comme l'unique centre où s'élaborent et d'où s'irradient les éternels moments de l'absolue vérité qui ne se réalise qu'en tant qu'esprit". Ibid.

7. Jean Bouthillette, le Canadien français et son double, Montréal, L'Hexagone, 1972.

8. «S'assimiler de fait, c'est mourir à soi pour renaître dans l'Autre», ou plutôt mourir dans l'Autre. (Ibid., p. 50)

9. Les citations qui ont trait au "regard aliéné» constituent le véritable leitmotif du livre de J. Bouthillette. Voici les plus marquantes: aL'identité canadienne est un miroir qui nous renvoie l'image de l'Autre quand nous nous y regardons." ( $p$. 47) "Faisant nôtre le regard que l'Anglais pose sur nous, nous avons perdu notre image, qui a pris le chemin de l'exil intérieur. » (p. 48) «Où que nous regardions, infailliblement nous rencontrons l'Autre - en l'occurence l'Anglais -, dont le regard trouble notre propre regard." (p. 15) “Faussée la relation à l'Anglais, c'est à sa racine psychique que l'en-soi canadien-français s'est faussé. Nous portons sur nous-mêmes et sur le monde un regard schizophrénique." (p. 50)

10. Dans Points de fuite, Montréal, Le Cercle du livre de France, 1971, p. 52.

11. Le 14 octobre 1967 dans la revue Perspectives. 